\title{
Treatment of adult brainstem glioma with combined antiangiogenic therapy: a case report and literature review
}

This article was published in the following Dove Medical Press journal:

OncoTargets and Therapy

\section{Dongdong Yu' \\ Guang Han ${ }^{2}$ \\ Huali Liu $^{3}$ \\ Likun $\mathrm{Gao}^{4}$ \\ Vivek Verma ${ }^{5}$}

'Department of Radiation Oncology, Renmin Hospital of Wuhan University, Wuhan, Hubei Province, P.R. China;

${ }^{2}$ Department of Radiation Oncology, Hubei Cancer Hospital, Wuhan, Hubei Province, P.R. China; ${ }^{3}$ Department of Oncology, Renmin Hospital of Wuhan University, Wuhan, Hubei Province, P.R. China; ${ }^{4}$ Department of Pathology, Renmin Hospital of Wuhan University, Wuhan, Hubei Province, P.R. China; ${ }^{5}$ Department of Radiation Oncology, Allegheny General Hospital, Pittsburgh, PA, USA
Correspondence: Guang Han Department of Radiation Oncology, Hubei Cancer Hospital, II6 Zhuodaoquan South Road, Wuhan 430079, Hubei Province, P.R. China Tel +86 I $3886048 \mid 78$

Fax +86 2787670275 Email hg7913@hotmail.com

\begin{abstract}
Adult brainstem gliomas belong to a rare and heterogeneous group of brain tumors. The overall prognosis is poor; therapeutic options are limited, given the resistance to radiotherapy and the unclear role of chemotherapy/antiangiogenic therapy. Apatinib, a tyrosine kinase inhibitor that selectively inhibits the vascular endothelial growth factor receptor and mildly inhibits c-Kit, PDGFR- $\beta$, RET, and c-SRC, has been reported to show efficacy among some patients with malignant supratentorial gliomas. However, its effect on brainstem glioma has not been reported so far. Herein, a 66-year-old man with brainstem anaplastic astrocytoma isocitrate dehydrogenase (IDH) wild type was treated initially with combined radiotherapy, temozolomide, and apatinib. The patient achieved a complete response by MRI and continues to have an ongoing progression-free survival of over 8 months. To our knowledge, this is the first case report using apatinib to treat brainstem IDH wild-type anaplastic astrocytoma, displaying an excellent outcome. We also summarize cases of adult brainstem glioma treated with antiangiogenic therapy. Experiences using various regimens may improve understanding of this rare disease, and thus help physicians to seek more effective treatments for these patients.
\end{abstract}

Keywords: VEGFR, antiangiogenic therapy, apatinib, brainstem glioma

\section{Introduction}

Adult brainstem gliomas (BSGs) are uncommon and constitute only $2 \%$ of all adult brain tumors; as such, they are a poorly characterized cohort with variable prognosis. ${ }^{1-3}$ The available treatment strategies for BSG include surgery, radiotherapy, chemotherapy, and/or biological treatment. However, owing to the rarity of BSG in adults, no large clinical trials have been conducted, and as such, standard strategies are still controversial. Radiotherapy is considered the cornerstone of adult BSG, ${ }^{4}$ while the role of chemotherapeutic and biological agents has not been well studied. Herein, we report an adult with isocitrate dehydrogenase (IDH) wild-type anaplastic astrocytoma (WHO grade III) who experienced a remarkable response to the combination of external-beam fractionated radiation, concomitant temozolomide (TMZ), and the small molecule tyrosine kinase inhibitor (TKI) apatinib. Additionally, cases of adult BSG treated with antiangiogenic therapy are discussed in a review of the literature.

\section{Ethics statement}

Ethics approval of this case report was granted by the Institutional Ethics Review Board of Renmin Hospital of Wuhan University. Written informed consent was obtained from the patient for publication of this case report and any accompanying images. Institutional approval was not required to publish this manuscript. 


\section{Case description}

\section{Clinical presentation}

A 66-year-old male was referred to the Department of Neurosurgery for 2 months of dizziness, weakness, and gait ataxia. Physical examination showed dysarthria and bilateral lower limb weakness with grade 2 muscle strength; the patient could not complete the heel-knee-tibia and left finger-nose-finger tests. The Karnofsky performance status (KPS) at diagnosis was 30.

T2-weighted (T2W) magnetic resonance imaging (MRI) showed a hyperintense signal alteration in the right side of the pons. Contrast-enhanced T1-weighted (T1W) MRI showed increased enhancement within the tumor. Three-dimensional arterial spin labeling (3D-ASL) showed increased cerebral blood flow (CBF) value in the right pons (Figure 1). A BSG was thus diagnosed radiologically.

Enhancing BSGs have a wide range of differential diagnoses on MRI, such as ependymoma, hemangioma, and metastasis. ${ }^{3}$ In order to rule out these image mimics, microsurgery was performed to obtain histologic diagnosis. The tumor was depicted by the surgeon as located in the pontine gray-white areas, ill-defined, and with a rich blood supply. Since resection was not possible, tissues were obtained for biopsy. No severe perioperative complications were observed. After operation, there was no improvement in neurological symptoms.

\section{Histologic examination}

H\&E staining showed features of anaplastic astrocytoma (WHO grade III) with increased cellularity, nuclear atypia, and mitotic activity. Immunohistochemical staining for GFAP and oligodendrocyte transcription factor were positive, further supporting the diagnosis. High proliferative activity was present, with $60 \%$ positive cells staining for Ki-67. Vascular endothelial growth factor receptor-1 (VEGFR-1) and vascular endothelial growth factor receptor-2 (VEGFR-2) were positive; the tumor was negative for stem cell factor receptor (c-kit, CD117) and H3K27M (Figure 2).

The MGMT promoter region was methylated, and loss of heterozygosity was present in chromosome $1 \mathrm{p}$ and absent in chromosome 19q; no mutations were found in IDH1R132, IDH2R172, TERTC228T, TERTC250T, BRAFV600E, H3F3AK27M, and HIST1H3BK27M (Table 1).

\section{Treatment}

More than 1 month after microsurgery, the patient was referred for further treatment. Considering the poor location, history of rapid progression, as well as surgical and histopathologic findings, we decided to administer radiotherapy with concomitant chemotherapy (TMZ) and antiangiogenic therapy (apatinib). The patient's hematologic, hepatic, renal, and cardiac functions were all within normal limits before commencing treatment.
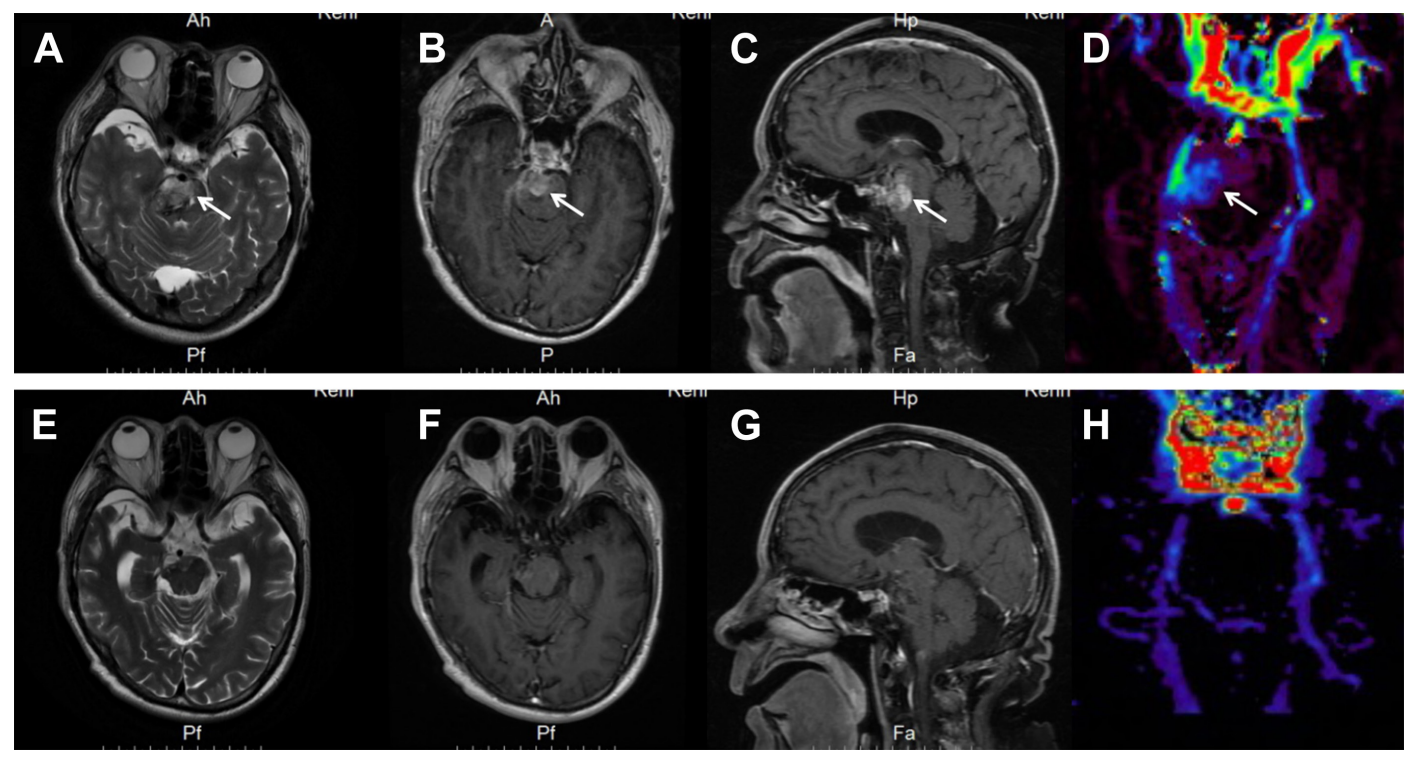

Figure I Brain MRI scans.

Notes: MRI before treatment (A-D): (A) axial T2W shows a hyperintense signal alteration in the right side of the pons; (B) axial TIW post-contrast; (C) sagittal TIW postcontrast; and (B and C) increased enhancement within the tumor. (D) 3D-ASL CBF shows increased CBF value in the right pons. MRI after treatment (E-H): (E) axial T2W shows a decreased hyperintense signal in the right side of the pons compared to pretreatment; (F) axial TIW post-contrast; (G) sagittal TIW post-contrast; and (F and $\mathbf{G})$ disappeared right pontine lesion. (H) 3D-ASL CBF shows decreased CBF value in the right pons compared to pretreatment. Abbreviations: 3D-ASL, three-dimensional arterial spin labelling; CBF, cerebral blood flow; TIW, TI weighted; T2W, T2 weighted. 

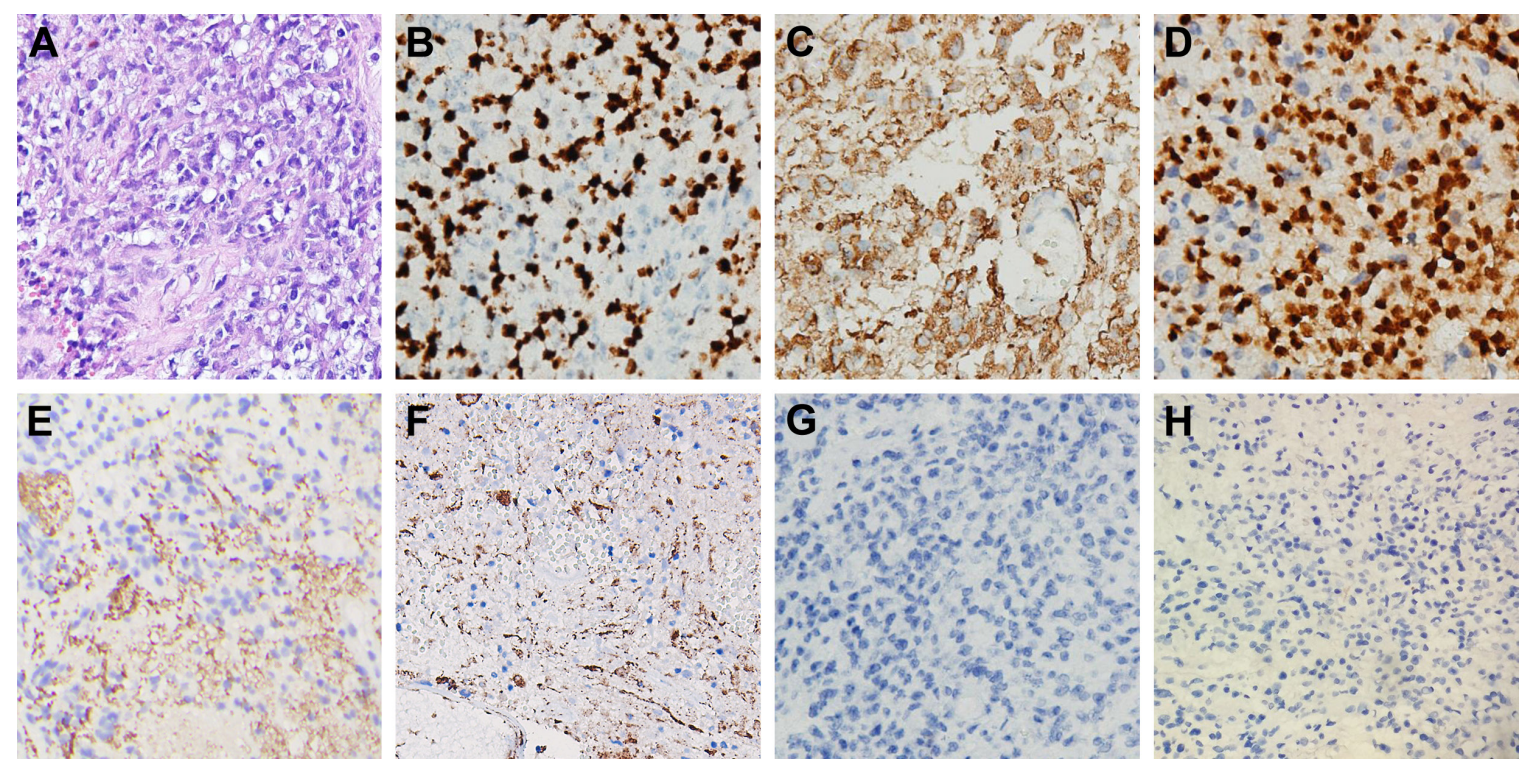

Figure 2 Histopathologic and immunohistochemical examinations of the biopsy tissue.

Notes: (A) H\&E stain showed features of anaplastic astrocytoma (WHO grade III) with increased cellularity, nuclear atypia, and mitotic activity. (B) MiBI (Ki-67) immunohistochemistry showed high proliferative activity with $60 \%$ positive cells. (C) GFAP immunohistochemistry. (D) Oligo-2 immunohistochemistry. (E) VEGFR-I immunohistochemistry. (F) VEGFR-2 immunohistochemistry. (G) CDII7 (c-kit). (H) H3K27M immunohistochemistry. Staining was positive for GFAP, oligo-2, VEGFRI, and VEGFR2 and negative for c-kit and H3K27M. Magnification 400x.

Abbreviation: VEGFR, vascular endothelial growth factor receptor.

From August 29 to October 11, 2017, the patient was treated with intensity-modulated radiation therapy (IMRT) and concomitant TMZ chemotherapy and apatinib-targeted therapy. The dose of IMRT was 56 Gy in 30 fractions, TMZ was $75 \mathrm{mg} / \mathrm{m}^{2} /$ day, and apatinib was $500 \mathrm{mg} /$ day. Mannitol and nutritional support were administered during the treatment period, and headaches disappeared after ten fractions, on September 8.

During the period of combined-modality therapy, the patient experienced grade 1 leukopenia and thrombocytopenia, without other adverse events as defined by the National Cancer Institute Common Terminology Criteria for Adverse Events (version 4.0). When the combinedmodality therapy got completed, on October 11, physical

Table I Results of molecular tests

\begin{tabular}{l|l}
\hline Item & Result \\
\hline MGMT promoter methylation & Positive \\
Loss of heterozygosity of chromosome IP & Positive \\
Loss of heterozygosity of chromosome I9q & Negative \\
IDHI RI32 mutation & Negative \\
IDH2 RI72 mutation & Negative \\
TERT C228T mutation & Negative \\
TERT C250T mutation & Negative \\
BRAF V600E mutation & Negative \\
H3F3A K27M mutation & Negative \\
HISTIH3B K27M mutation & Negative \\
\hline
\end{tabular}

examination showed grade 4 muscle strength of bilateral lower limbs, which was greatly improved over that prior to treatment. However, his dysarthria persisted. After the concurrent therapy, we recommended continuation of apatinib (500 mg daily) following treatment. Unfortunately, 2 weeks later (October 25), apatinib was discontinued due to financial reasons. The patient began to receive adjuvant TMZ on November 18, according to the 5-day schedule, every 28 days. The dose of TMZ was $150-200 \mathrm{mg} / \mathrm{m}^{2}$, and chemotherapy was continued if no hematologic toxicities occurred at the beginning of the next cycle, and a total of six cycles was planned.

The patient returned for reevaluation (after concurrent therapy) on November 16. At that time, the patient could walk slowly with assistance and dysarthria had ceased. The KPS was 60. T2W MRI showed a decreased hyperintense signal in the right side of the pons compared to pretreatment. T1W post-contrast MRI showed that the right pontine lesion had disappeared. 3D-ASL showed decreased CBF value in the right pons compared to pretreatment (Figure 1E-H). At the time of last follow-up, the patient was independent in all daily activities. MRI at the time (performed at outside facility) showed no signs of tumor recurrence. According to the Response Assessment in Neuro-Oncology criteria, he achieved a complete response (CR). ${ }^{5}$ 


\section{Discussion}

BSG in adults represents a heterogeneous group of tumors whose presentation and clinical course depend on pathologic and molecular features. Owing to the location of these tumors, biopsies are not routinely performed. An MRI-based radiological classification has been proposed to establish treatment strategies and to determine outcomes for 1) diffuse intrinsic low-grade gliomas, 2) enhancing malignant gliomas, 3) focal tectal gliomas, and 4) exophytic gliomas/ other subtypes.,6,7

Among the different subtypes above, enhancing malignant gliomas (WHO grade III-IV) carry the poorest prognosis. ${ }^{8} 9$ They (accounting for up to $30 \%$ of adult BSGs) comprise the majority of BSG in adults. Radiotherapy is reported to have limited efficacy, and only a small proportion of patients $(<13 \%)$ exhibit clinical and radiographic improvement after radiation. ${ }^{2}$ Even higher doses (up to 72 Gy) fail to improve efficacy and carry a higher risk of radiation-related complications. ${ }^{1}$ The role of chemotherapy in the treatment of enhancing malignant adult BSG remains unclear; TMZ or combined procarbazine, lomustine, and vincristine added to radiation does leads to clinical improvement in some patients, but grade 3 and 4 myelosuppression may also occur. ${ }^{10}$

We summarize published reports of histologically diagnosed BSG in Table $2 .^{4,8,11-15}$ In these reports, the median overall survival for brainstem glioblastoma ranges from 6 to 16 months, along with a wide range of 12-77 months for brainstem anaplastic astrocytoma. The KPS score, age, duration of symptoms, pontine location, and tumor grade impact survival. . $^{1,2,4,8,11-16}$ In general, efficacious therapies are limited in malignant BSG. Therefore, novel therapies are required to avoid deterioration of clinical symptoms and prolong survival.

Antiangiogenic therapy can normalize the structure and function of tumor vessels, resulting in improved drug delivery and a normalized tumor microenvironment. This effect may underlie the therapeutic benefit of combined antiangiogenic and cytotoxic therapies. ${ }^{17,18}$ In recent years, there have been several reports regarding antiangiogenic therapy, such as bevacizumab (an anti-vascular endothelial growth factor monoclonal antibody, which has been intensively studied in recurrent glioblastoma), as an effective salvage therapy for progressive BSG. ${ }^{19}$ After administering bevacizumab, an improvement in clinical conditions has been shown, along with satisfactory radiologic responses and a progression-free survival (PFS) up to 2 years. ${ }^{20-24}$ However, the efficacy of first-line antiangiogenic therapy for adult BSG is unknown (Table 3). The cases we summarized include eleven patients with BSG, five diagnosed radiologically and the remainder with histologic diagnosis. For histologically diagnosed BSG, PFS with bevacizumab ranges from 4 to 21 months; for four

Table 2 Survival data of histologically diagnosed brainstem gliomas in adults

\begin{tabular}{|c|c|c|c|c|c|}
\hline References & $\begin{array}{l}\text { Tumor } \\
\text { histology }\end{array}$ & $\begin{array}{l}\text { No. of } \\
\text { patients }\end{array}$ & Therapy & $\begin{array}{l}\text { mPFS } \\
\text { (months) }\end{array}$ & $\begin{array}{l}\text { mOS } \\
\text { (months) }\end{array}$ \\
\hline $\begin{array}{l}\text { Babu et al }(2013)^{12} \\
n=7 \\
\text { Babu et al }(2014)^{13} \\
n=34 \\
\text { Hundsberger et al }(2014)^{11} \\
n=21 \\
\text { Dey et al }(2014)^{14} \\
n=240 \\
\text { Reithmeier et al }(2014)^{4} \\
n=104^{b} \\
\begin{array}{l}\text { Theeler et al }(20 \mid 5)^{15} \\
n=143^{\text {a }}\end{array}\end{array}$ & $\begin{array}{l}\text { WHO grade I } \\
\text { WHO grade II } \\
\text { WHO grade III } \\
\text { WHO grade IV } \\
\text { AA } \\
\text { GB } \\
\text { AA } \\
\text { GB } \\
\text { LG-BSG } \\
\text { HG-BSG } \\
\text { AA } \\
\text { GB } \\
\text { WHO grade I } \\
\text { WHO grade II } \\
\text { WHO grade III } \\
\text { WHO grade IV } \\
\text { DA } \\
\text { AA } \\
\text { GB }\end{array}$ & $\begin{array}{l}16 \\
15 \\
12 \\
3 \\
3 \\
4 \\
22 \\
12 \\
8 \\
13 \\
75 \\
165 \\
16 \\
31 \\
42 \\
14 \\
4 \\
18 \\
21\end{array}$ & $\begin{array}{l}\text { Radiation, chemotherapy (PCV, lomustine, } \\
\text { vincristine, carboplatin, carmustine, } \\
\text { temozolomide, irinotecan) } \\
\text { Radiotherapy and concurrent temozolomide, } \\
\text { other salvage chemotherapy } \\
\text { Radiotherapy, temozolomide, other salvage } \\
\text { chemotherapy, and/or bevacizumab } \\
\text { Radiotherapy and/or chemotherapy, supportive care } \\
\text { Radiation, data regarding chemotherapy, and other } \\
\text { treatment regimens NA } \\
\text { Radiation, chemotherapy, interstitial radiosurgery } \\
\text { (with I-I25 seeds), combined radiation and } \\
\text { chemotherapy, supportive care } \\
\text { Radiation, chemotherapy (temozolomide, } \\
\text { cytotoxic chemotherapy, and/or bevacizumab) }\end{array}$ & $\begin{array}{l}44 \\
48 \\
10 \\
10 \\
6.7 \\
(A A+G B) \\
\text { NA } \\
\text { NA } \\
24.1 \\
5.8 \\
\text { NA } \\
\text { NA } \\
\text { NA } \\
\text { NA } \\
\text { NA } \\
\text { NA } \\
\text { NA } \\
\text { NA } \\
\text { NA }\end{array}$ & $\begin{array}{l}83 \\
168 \\
17 \\
16 \\
13.5 \\
(\mathrm{AA}+\mathrm{GB}) \\
77 \\
12.1 \\
30.5 \\
11.5 \\
12 \\
6 \\
\mathrm{NA} \\
26.2 \\
12.9 \\
9.8 \\
77 \\
21.1 \\
14.8\end{array}$ \\
\hline
\end{tabular}

Notes: a The study contained patients who were diagnosed radiologically. ${ }^{\text {In }}$ one patient, grading was not performed.

Abbreviations: AA, anaplastic astrocytoma; DA, diffuse astrocytoma; GB, glioblastoma; HG-BSG, high-grade brainstem glioma; LG-BSG, low-grade brainstem glioma; mOS, median overall survival; mPFS, median progression-free survival; NA, not available; PCV, procarbazine, lomustine, and vincristine. 


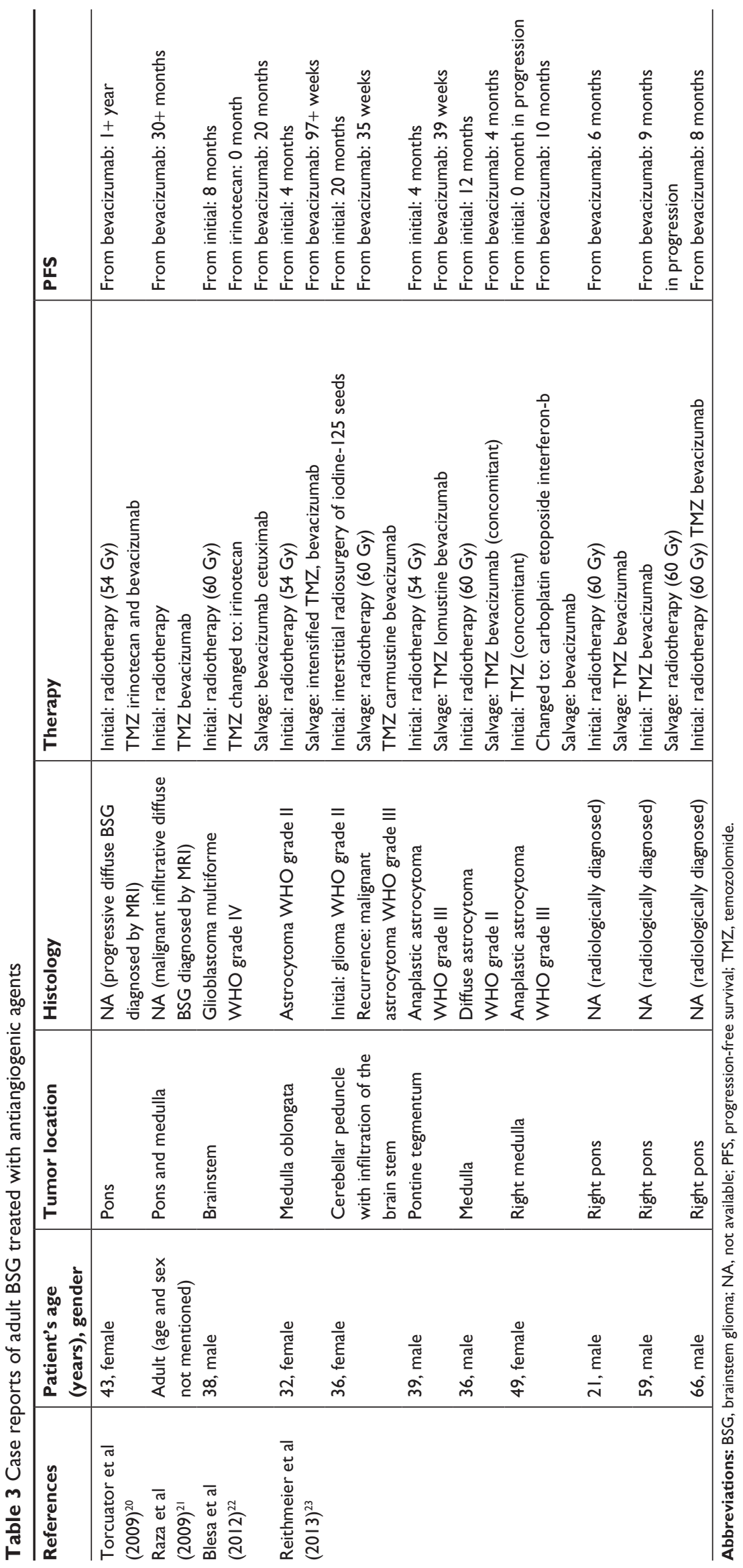


patients who were given bevacizumab as salvage therapy, the PFS was even much longer than the PFS in the initial radiotherapy setting. ${ }^{22,23}$

Despite the presence of the following poor prognostic factors such as declining performance status, age, tumor location, histology, as well as the limited efficacy of radiotherapy or chemotherapy for malignant BSG, we made the decision to treat the patient with combined-modality management. Because the tumor contained a rich blood supply as determined by the surgeon, and 3D-ASL showed increased $\mathrm{CBF}$, we surmised that combined antiangiogenic therapy may achieve a meaningful benefit.

Receptor-specific antibodies and small molecular TKIs have been developed for antiangiogenic therapy. Because bevacizumab was most commonly used and reported to be effective in combined antiangiogenic therapy of BSG, ${ }^{20-24}$ bevacizumab was recommended initially, but the patient refused for financial reasons. Then, we suggested consideration of TKIs (such as sorafenib, pazopanib, sunitinib, or apatinib), which target the vascular endothelial growth factor receptor (VEGFR). ${ }^{25-29}$ Owing to the rarity of BSG in adults, reported experience of TKIs in this disease was also rare, and we were not able to find guidelines for our patient. In order to choose the best agent for our patient, we examined the expression of VEGFR-1 and VEGFR-2. Immunohistochemistry showed that both VEGFR-1 and VEGFR-2 were positive. As VEGFR-2 plays an important role in angiogenesis, agents targeting VEGFR-2 should be theoretically helpful for tumor suppression. Animal experiments showed that the value of $\mathrm{IC}_{50}$ to VEGFR-2 for apatinib was $2 \mathrm{nM}$, while the values for sorafenib, pazopanib, and sunitinib were 90, 30, and $10 \mathrm{nM}$, respectively, ${ }^{30}$ which indicated the high specificity of VEGFR-2 for apatinib. Apatinib can highly selectively compete for the ATP-binding site of intracellular VEGFR-2 to block downstream signal transduction and inhibit tumor angiogenesis Moreover, besides antiangiogenic effects, apatinib can also mildly inhibit c-Kit, c-SRC, PDGFR- $\beta$, and MET, exerting antitumor effects directly. ${ }^{28,29}$ A case series reported that apatinib had outstanding efficacy for refractory malignant glioma; one patient achieved near CR as evaluated by MRI and had an overall survival of 27 weeks, and another achieved partial response and had a PFS of 12 months. ${ }^{31}$ Another pilot study of apatinib plus irinotecan in patients with recurrent high-grade glioma showed an objective response rate of 55\% (5/9), disease control rate of 78\% (7/9), and median PFS of 8.3 months. The most common adverse events were gastrointestinal reaction (31.8\%), hypertension $(22.7 \%)$, and myelosuppression (18.0\%). ${ }^{32}$ In our case, positive VEGFR-2 staining observed on the histologic specimen, along with lower costs and ease of administration, led us to consider apatinib over intravenous bevacizumab and other TKIs.

To our knowledge, this is the first known case in which a malignant BSG was managed with apatinib and chemoradiation. The patient achieved a radiologic CR at 4 weeks after treatment. His KPS was 70 at last follow-up, the PFS was over 8 months, and only grade 1 leukopenia and thrombocytopenia were observed; other toxicities for apatinib, such as hypertension, proteinuria, or hand-foot skin reaction, were not developed in our patient. However, among cases that we summarized above in which malignant BSGs were managed with bevacizumab and chemoradiation, ${ }^{20-24}$ only one patient who was diagnosed by MRI showed disappeared contrast enhancement in the tumor lesion region after treatment. ${ }^{21}$ Compared to the therapeutic effect with bevacizumab, the treatment response with apatinib in our case was encouraging; although it is only one case, it provides an additional treatment option for malignant BSG.

H3K27M mutations are frequently observed in adult $\mathrm{BSG}$ and tend to be associated with a poorer prognosis. ${ }^{33} \mathrm{~A}$ publication indicated that the H3K27M mutation defined a distinct subgroup of IDH wild-type gliomas characterized by a constant midline location, low rate of MGMT promoter methylation, and poor prognosis in gliomas. ${ }^{34}$ In this patient, who was H3K27M negative, we also performed molecular analysis of histone $\mathrm{H} 3$ mutations (negative for $H 3 F 3 A \mathrm{~K} 27 \mathrm{M}$ and HIST1H3BK27M). Potentially, this may be a contributing factor to the excellent outcome of this patient.

In this case, owing to the limited biopsy tissue (except for c-Kit), we did not evaluate the expression of other potential target genes, such as PDGFR- $\beta$, RET, and c-SRC, which might also contribute to the potential therapeutic effect of apatinib. We consider apatinib to not just be an antiangiogenic agent, but it may also act on the tumor cells directly in this case, especially in the absence of an intact blood-brain barrier, as evidenced by the contrast enhancement on MRI.

\section{Conclusion}

To our knowledge, this is the first case report using apatinib to treat brainstem IDH wild-type anaplastic astrocytoma with an excellent outcome, and the toxicities are tolerable. The case describes a novel regimen for clinicians encountering this rare and difficult-to-treat brain tumor.

\section{Acknowledgments}

This research received financial support from the Chinese Society of Clinical Oncology Foundation (grant no. Y-HR2016-44) and the Hubei Province Natural Science Foundation (no. 2016CFC737). 


\section{Disclosure}

All of the authors declare that the article content was composed in the absence of any commercial or financial relationships that could be construed as a potential conflict of interest.

\section{References}

1. Landolfi JC, Thaler HT, DeAngelis LM. Adult brainstem gliomas. Neurology. 1998;51(4):1136-1139.

2. Guillamo JS, Monjour A, Taillandier L, et al. Brainstem gliomas in adults: prognostic factors and classification. Brain. 2001;124(Pt 12): 2528-2539.

3. Purohit B, Kamli AA, Kollias SS. Imaging of adult brainstem gliomas. Eur J Radiol. 2015;84(4):709-720.

4. Reithmeier T, Kuzeawu A, Hentschel B, et al. Retrospective analysis of 104 histologically proven adult brainstem gliomas: clinical symptoms, therapeutic approaches and prognostic factors. BMC Cancer. 2014; 14(1):115-122.

5. Wen PY, MacDonald DR, Reardon DA, et al. Updated response assessment criteria for high-grade gliomas: response assessment in neuro-oncology working group. J Clin Oncol. 2010;28(11):1963-1972.

6. Reyes-Botero G, Mokhtari K, Martin-Duverneuil N, Delattre JY, LaigleDonadey F. Adult brainstem gliomas. Oncologist. 2012;17(3):388-397.

7. Ramos A, Hilario A, Lagares A, et al. Brainstem gliomas. Semin Ultrasound CT MR. 2013;34(2):104-112.

8. Kesari S, Kim RS, Markos V, et al. Prognostic factors in adult brainstem gliomas: a multicenter, retrospective analysis of 101 cases. J Neurooncol. 2008;88(2):175-183.

9. Rineer J, Schreiber D, Choi K, Rotman M. Characterization and outcomes of infratentorial malignant glioma: a population-based study using the surveillance epidemiology and end-results database. Radiother Oncol. 2010;95(3):321-326.

10. Salmaggi A, Fariselli L, Milanesi I, et al. Natural history and management of brainstem gliomas in adults. A retrospective Italian study. J Neurol. 2008;255(2):171-177.

11. Hundsberger T, Tonder M, Hottinger A, et al. Clinical management and outcome of histologically verified adult brainstem gliomas in Switzerland: a retrospective analysis of 21 patients. J Neurooncol. 2014;118(2):321-328.

12. Babu R, Kranz PG, Karikari IO, Friedman AH, Adamson C. Clinical characteristics and treatment of malignant brainstem gliomas in elderly patients. J Clin Neurosci. 2013;20(10):1382-1386.

13. Babu R, Kranz PG, Agarwal V, et al. Malignant brainstem gliomas in adults: clinicopathological characteristics and prognostic factors. J Neurooncol. 2014;119(1):177-185.

14. Dey M, Lin Y, Melkonian S, Lam S. Prognostic factors and survival in primary adult high grade brainstem astrocytoma: a population based study from 1973-2008. J Clin Neurosci. 2014;21(8):1298-1303.

15. Theeler BJ, Ellezam B, Melguizo-Gavilanes I, et al. Adult brainstem gliomas: correlation of clinical and molecular features. J Neurol Sci. 2015;353(1-2):92-97.

16. Grigsby PW, Garcia DM, Simpson JR, Fineberg BB, Schwartz HG. Prognostic factors and results of therapy for adult thalamic and brainstem tumors. Cancer. 1989;63(11):2124-2129.

OncoTargets and Therapy

\section{Publish your work in this journal}

OncoTargets and Therapy is an international, peer-reviewed, open access journal focusing on the pathological basis of all cancers, potential targets for therapy and treatment protocols employed to improve the management of cancer patients. The journal also focuses on the impact of management programs and new therapeutic agents and protocols on

Submit your manuscript here: http://www.dovepress.com/oncotargets-and-therapy-journal
17. Jain RK. Antiangiogenic therapy for cancer: current and emerging concepts. Oncology. 2005;19(4 Suppl 3):7-16.

18. Jain RK, di Tomaso E, Duda DG, et al. Angiogenesis in brain tumours. Nat Rev Neurosci. 2007;8(8):610-622.

19. Kreisl TN, Kim L, Moore K, et al. Phase II trial of single-agent bevacizumab followed by bevacizumab plus irinotecan at tumor progression in recurrent glioblastoma. J Clin Oncol. 2009;27(5):740-745.

20. Torcuator R, Zuniga R, Loutfi R, Mikkelsen T. Bevacizumab and irinotecan treatment for progressive diffuse brainstem glioma: case report. J Neurooncol. 2009;93(3):409-412.

21. Raza S, Donach M. Bevacizumab in adult malignant brainstem gliomas. J Neurooncol. 2009;95(2):299-300.

22. Blesa JM, Mollá SB, Esparcia MF, et al. Durable complete remission of a brainstem glioma treated with a combination of bevacizumab and cetuximab. Case Rep Oncol. 2012;5(3):676-681.

23. Reithmeier T, Lopez WO, Spehl TS, et al. Bevacizumab as salvage therapy for progressive brain stem gliomas. Clin Neurol Neurosurg. 2013; 115(2):165-169.

24. Moriya S, Ohba S, Adachi K, et al. A retrospective study of bevacizumab for treatment of brainstem glioma with malignant features. J Clin Neurosci. 2018;47:228-233.

25. Wedge SR, Ogilvie DJ, Dukes M, et al. ZD4190: an orally active inhibitor of vascular endothelial growth factor signaling with broad-spectrum antitumor efficacy. Cancer Res. 2000;60(4):970-975.

26. Wood JM, Bold G, Buchdunger E, et al. PTK787/ZK 222584, a novel and potent inhibitor of vascular endothelial growth factor receptor tyrosine kinases, impairs vascular endothelial growth factor-induced responses and tumor growth after oral administration. Cancer Res. 2000; 60(8):2178-2189.

27. Clark JW, Eder JP, Ryan D, Lathia C, Lenz HJ. Safety and pharmacokinetics of the dual action Raf kinase and vascular endothelial growth factor receptor inhibitor, Bay 43-9006, in patients with advanced, refractory solid tumors. Clin Cancer Res. 2005;11(15):5472-5480.

28. Tong XZ, Wang F, Liang S, et al. Apatinib (YN968D1) enhances the efficacy of conventional chemotherapeutical drugs in side population cells and ABCB1-overexpressing leukemia cells. Biochem Pharmacol. 2012; 83(5):586-597.

29. Geng R, Li J. Apatinib for the treatment of gastric cancer. Expert Opin Pharmacother. 2015;16(1):117-122.

30. Cowey CL, Sonpavde G, Hutson TE. New advancements and developments in treatment of renal cell carcinoma: focus on pazopanib. Onco Targets Ther. 2009;3(4):147-155.

31. Zhang H, Chen F, Wang Z, Wu S, et al. Successful treatment with apatinib for refractory recurrent malignant gliomas: a case series. Onco Targets Ther. 2017;10:837-845.

32. Wang L, Liang L, Yang T, et al. A pilot clinical study of apatinib plus irinotecan in patients with recurrent high-grade glioma: clinical trial/ experimental study. Medicine. 2017;96(49):e9053.

33. Feng J, Hao S, Pan C, et al. The H3.3 K27M mutation results in a poorer prognosis in brainstem gliomas than thalamic gliomas in adults. Hum Pathol. 2015;46(11):1626-1632.

34. Meyronet D, Esteban-Mader M, Bonnet C, et al. Characteristics of H3 K27M-mutant gliomas in adults. Neuro Oncol. 2017;19(8):1127-1134.

patient perspectives such as quality of life, adherence and satisfaction. The manuscript management system is completely online and includes a very quick and fair peer-review system, which is all easy to use. Visit http://www.dovepress.com/testimonials.php to read real quotes from published authors.

\section{Dovepress}

